\title{
Statistics of Electrical Dispersion Compensator Penalties of 10-Gb/s Multimode Fiber Links With Offset Connectors
}

\author{
Yu Sun, Senior Member, IEEE, P. Hallemeier, Member, IEEE, H. Ereifej, Member, IEEE, \\ O. V. Sinkin, Member, IEEE, B. S. Marks, Member, IEEE, and C. R. Menyuk, Fellow, IEEE
}

\begin{abstract}
In this letter, we investigate the penalty after a 10-Gb/s Ethernet signal is detected and processed. We study the statistics of ideal electrical dispersion-compensation (EDC) performance, measured by the penalty of an ideal decision feedback equalizer (PIE-D), of 300-m multimode fiber links to determine the theoretically best possible EDC performance. We calculate the channel responses using two methods: the individual-mode method and the average-mode method. We find a strong dependence of the PIE-D value on the channel model that is used, especially when there is a connector with a large offset in the link. In system design, one should choose a suitable channel model, taking into account the length of the fiber, the magnitude of offset, and the percentage of coverage.
\end{abstract}

Index Terms-Decision feedback equalizers, electrical dispersion compensator, mode delays, multimode transmission line, optical transmission.

\section{INTRODUCTION}

$\mathbf{E}$ LECTRICAL dispersion compensation (EDC) has drawn increasing attention in multimode fiber (MMF) link applications. The IEEE 802.3aq taskforce, which was tasked with standardizing the 10GBASE-LRM (long reach multimode) link for $10-\mathrm{Gb} / \mathrm{s}$ data transmission over legacy MMF using the existing 10G BASE-R PCS (physical coding sublayer) serial transmission, voted to make EDC the primary means of channel equalization [1]. EDC devices designed for $10-\mathrm{Gb} / \mathrm{s}$ communication links over fiber-distributed-data-interface (FDDI)-grade legacy MMF have been demonstrated [2].

In our study we use the penalty of an ideal decision feedback equalizer (PIE-D) with an infinite number of taps to determine the theoretical best-case EDC performance [3]. In order to calculate the penalty of such an equalizer, the optical channel response must be known.

In our previous work, we shown that simulated results using the individual-mode method closely resembled the axial asymmetric in the channel response, observed in a $300 \mathrm{~m} \mathrm{MMF} \mathrm{link}$ when the connector offset was varied [4]. While those using the average-mode method [5] did not.

Manuscript received August 24, 2006; revised January 30, 2007.

Y. Sun, P. Hallemeier, and H. Ereifej are with Optium Corporation, Horsham, PA 19044 USA (e-mail: ysun@optium.com; phallemeier@optium.com; heider@optium.com).

O. V. Sinkin, B. S. Marks, and C. R. Menyuk are with the Department of Computer Science and Electrical Engineering, University of Maryland Baltimore County, Baltimore, MD 21250 USA (e-mail: oleg.sinkin@umbc.edu; marks@umbc.edu; menyuk@umbc.edu).

Color versions of one or more of the figures in this letter are available online at http://ieeexplore.ieee.org.

Digital Object Identifier 10.1109/LPT.2007.894356
Different channel responses may cause the same EDC penalty. Hence, our earlier results do not demonstrate the necessity of using the individual-mode model in practice. One should therefore determine whether these two channel models predict any significant difference in the system penalty after the signal is received and processed.

In this letter, we study the statistics of the PIE-D. We used both methods to simulate the channel responses of 300-m multimode links with offset connectors. A set of 5000 FDDI grade MMFs [6] was simulated and the penalty of the equalizer for every fiber was calculated. We found a significant difference between the predictions of the two methods when there are large connector offsets in the link. The penalty difference is $0.7 \mathrm{~dB}$ for a $7-\mu \mathrm{m}$ offset when the coverage is $99 \%$ for both the center launch and the offset launch and less than $0.1 \mathrm{~dB}$ for a $5-\mu \mathrm{m}$ offset when the coverage is $99 \%$.

\section{REVIEW OF PREVIOUS WORK}

The effect of an axis offset in a connection is to redistribute light from each mode of the input (launching) fiber among the modes of the receiving fiber [7]. In our study, we did not consider the short fiber between the main fiber and the receiver, since it has little influence to the channel response. With an initial connection offset in the first fiber, the individual-mode method predicts asymmetric speckle pattern at the end of L1, in agreement with experiments [8], while the average-mode method predicts a symmetric speckle pattern.

In weakly index-guided fibers, the modes possessing similar group velocities are often lumped together into degenerate mode groups, and the delay of each mode group is denoted as the mode group delay [9]. Both methods properly account for the propagation delay in both L1 and the main fiber based on the fiber index profile. The first 18 mode groups are considered [10]. The mode group delay is used in the average-mode method, and the individual mode delays are used in the individual-mode method.

Fig. 1 shows the MMF link used in the experiment, consisting of two fibers with a length of 70 (fiber L1) and $230 \mathrm{~m}$ (main fiber), respectively. Due to the central defect, the MMF link is more challenging for a central launch. We injected $10-\mathrm{Gb} / \mathrm{s}$ nonreturn-to-zero (NRZ) signals generated by a linearly polarized 1550-nm distributed feedback laser and a $\mathrm{LiNbO}_{3}$ modulator through a single-mode fiber using a central launch. At the operating wavelength, the chromatic dispersion is negligible. We used the data pattern 000000001 and a $2^{7}-1$ pseudorandom bit string to measure the output pulse shape and the eye pattern at the receiver, respectively, using a digital sampling scope. We 


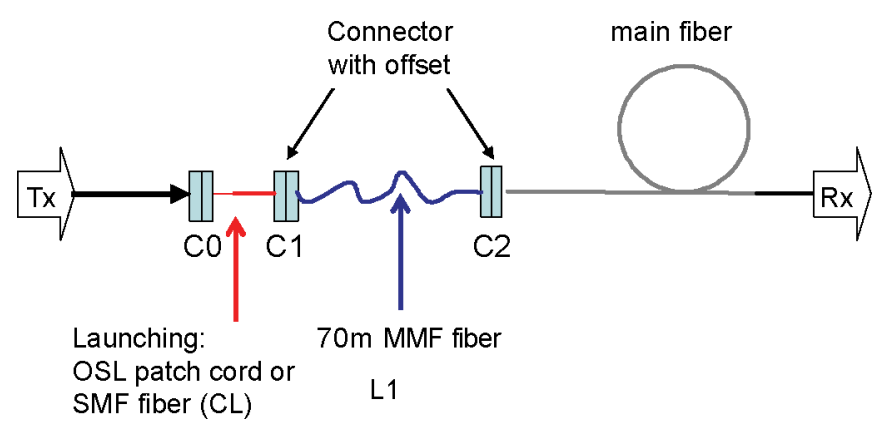

Fig. 1. Experimental setup.
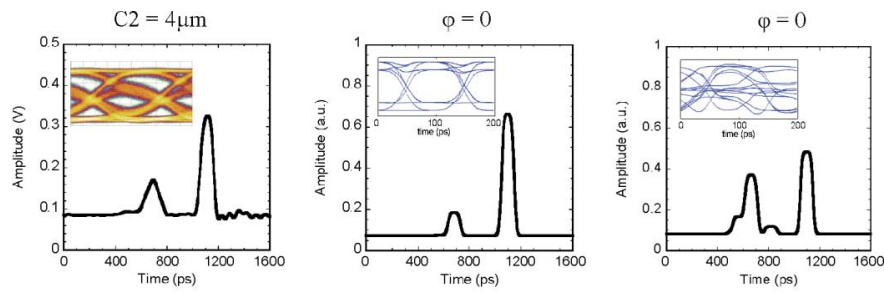

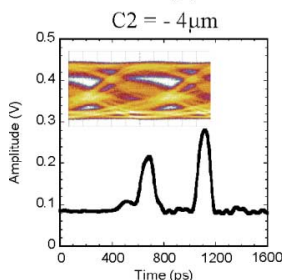

(a)

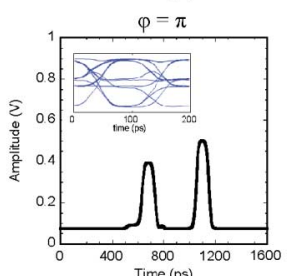

(b)

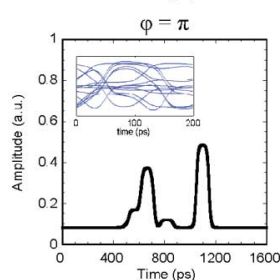

(c)
Fig. 2. Experimental and simulated results. (a) Experimental pulse shapes and the corresponding eye diagrams after a 300-m MMF link with a 3- $\mu \mathrm{m}$ offset at the input and $\pm 4-\mu \mathrm{m}$ offset at connector C2. (b) Simulated pulse shapes and the corresponding eye diagrams after 300-m MMF link with a 3- $\mu \mathrm{m}$ offset at the input and 4- $\mu \mathrm{m}$ offset at $\mathrm{C} 2$ with $\varphi=0, \pi$, respectively, using the individualmode method. (c) Simulated pulse shapes and the corresponding eye diagrams after 300-m MMF link with a 3- $\mu \mathrm{m}$ offset at the input and $4-\mu \mathrm{m}$ offset at $\mathrm{C} 2$ with $\varphi=0, \pi$, respectively, using the average-mode method.

considered 3 and $4 \mu \mathrm{m}$ for the offsets of $\mathrm{C} 1$ and $\mathrm{C} 2$, respectively, since the connector offset of a single-mode connector is usually lower than that of its multimode counterpart [11].

We moved the single-mode fiber $3 \mu \mathrm{m}$ vertically from the center of L1, so that two mode groups were excited. As we shifted the alignment of L1 to the main fiber center from 4 to $-4 \mu \mathrm{m}$. The power in the two mode groups varied accordingly, so that the eye diagrams at the receiver varied from being open to being completely closed, as shown in Fig. 2(a).

The index profile of the MMF with a $62.5-\mu \mathrm{m}$ diameter was simulated using a power-law index profile with a power-law value of 1.89 [12]. The indexes of the fiber core and cladding are 1.500 and 1.474 , respectively. We modeled the center defect as a Gaussian-shaped index change with a $3-\mu \mathrm{m}$ full-width at half-maximum (FWHM) and a maximum dip of 0.003 to match the measured differential group delay. We used a $7-\mu \mathrm{m}$ FWHM Gaussian beam to model the output field profile from a single-mode fiber and a 10-Gb/s NRZ pulse with a 30-ps rise time was injected into the link.

Simulated results are shown in Fig. 2(b) and (c). The simulation results using the individual-mode method agreed well with experimental results, while those using the average-mode method did not. Although these results are suggestive, they do

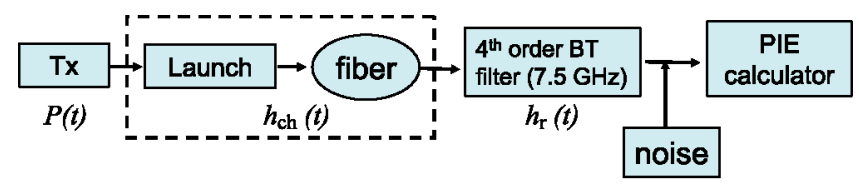

Fig. 3. Setup of the PIE-D statistics simulation.

not address the question of the greatest practical importance. In system design, what is important is the penalty after the signal is received and processed. Thus, one should determine whether these two channel models predict any significant difference in the system penalty.

\section{StATistics OF EDC PERFORMANCE}

We study the statistics of EDC penalties of 300-m multimode links with offset connectors and FDDI graded MMFs. In this study, we employ the PIE-D with an infinite number of taps to find the baseline for the best theoretically possible EDC performance. This baseline is independent of device designs.

In this study, we use 5000 sets of 18 relative delay values, which represent the DMD distribution of installed FDDI grade fiber base [5] with nonideal fibers profiles. With nonideal fiber profiles, the mode delays within each mode group differ. However, these nonidealities are not large enough in practice to change the channel response of $300-\mathrm{m} 10-\mathrm{Gb} / \mathrm{s}$ link significantly if 18 mode groups are considered [10]. Hence, we ignored fiber nonidealities in our study, so that the individual mode delays of each mode in a modal group are the same. The observed difference in performance between the average-mode method and the individual-mode method is entirely due to the unequal excitation of different modes in the modal groups.

The mode power distribution launched to the fiber is numerically computed by an overlap integral of the input Gaussian beam and the fiber modes of a truncated parabolic index profile. We use both the average-mode method and the individual-mode method to calculate the effect of the offset connector.

The simulation setup is shown in Fig. 3. The transmitter generates 10-Gb/s NRZ pulse with a 47.1-ps rising time, which is typical for a directly modulated laser. A fourth-order Bessel-Thompson filter with a 7.5-GHz FWHM is used to represent the receiver response. Two offset connectors are in the link, shown as $\mathrm{C} 1$ and $\mathrm{C} 2$ in Fig. 1. We set the fiber length between $\mathrm{C} 1$ and $\mathrm{C} 2$ to be $10 \mathrm{~m}$. The parameters are consistent with the system setup adopted by the IEEE 802.3 aq task force [13]. The total length of the link is $300 \mathrm{~m}$. The composite channel response is the convolution of the input pulse $p(t)$, response of the launch condition and the fiber link $h_{\mathrm{ch}}(t)$, and the receiver response $h_{r}(t)$, written as

$$
h(t)=p(t) * h_{\mathrm{ch}}(t) * h_{r}(t) .
$$

The composite channel response in the frequency domain can be obtained through Fourier transform (FT)

$$
H_{a}(f)=\mathrm{FT}[h(t)] .
$$

Gaussian white noise with a variance given by $\sigma^{2}=$ $3.16 \mathrm{~mW} / \mathrm{Hz}$ is added after the receiver, so that with a $6-\mathrm{dB}$ dispersion penalty, the bit-error ratio is $10^{-12}$ [14]. 


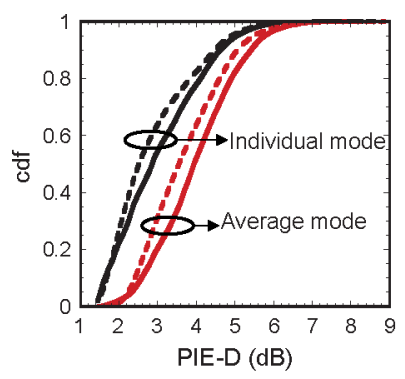

(a)

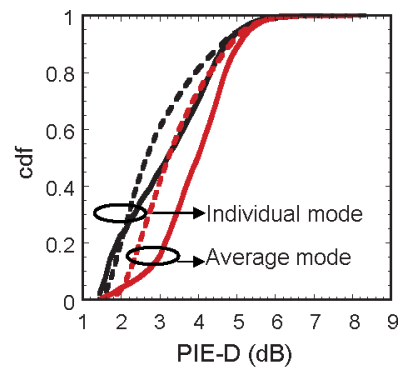

(b)
Fig. 4. Comparison of the cdfs of PIE-D after a 300-m MMF link with two offset connectors, using the individual-mode method and the average-mode method. The dashed lines correspond to an offset launch and the solid lines correspond to a central launch. (a) Both connectors have a 7- $\mu \mathrm{m}$ offset; (b) both connectors have a $5-\mu \mathrm{m}$ offset.

The PIE-D is defined as [14]

$$
\begin{aligned}
\mathrm{DFE} & =\exp \left[-2 T \int_{0}^{\frac{1}{2 T}} \ln \left(\frac{1}{T}\left|H_{a}(f)\right|^{2}+\sigma^{2}\right) d f\right] \\
\mathrm{PIE}-\mathrm{D} & =\frac{1}{2} \times 10 \log (\mathrm{DFE})
\end{aligned}
$$

where $T$ is the bit period.

The optical channel requires two main inputs: the launching condition and the fiber parameters. In this study, we focus on a single-mode Gaussian beam with an FWHM of $7 \mu \mathrm{m}$. In order to reflect the commonly used restricted launch condition, we investigate two cases: 1) a central launch, which only excites the lower order modes and 2) an offset launch, which shifts the input beam $17 \mu \mathrm{m}$ away from the fiber center.

When the average-mode method is used, since the output profile from the first fiber is axially symmetric, we only consider the magnitude of the offset at the connectors. The fiber impulse response is a superposition of impulse responses of each mode group, weighted by the mode power distribution with which the fiber is excited [5].

When the individual-mode method is used, we consider not only the magnitude of the offset but the orientations. We rotate the offset connector from 0 to $2 \pi$ with 50 even steps.

We consider two cases of offset combination: $\mathrm{C} 1$ and $\mathrm{C} 2$ both have a 5- $\mu \mathrm{m}$ offset and a 7- $\mu \mathrm{m}$ offset, respectively. For each case, we also consider both the center launch and the offset launch. The cumulative distribution functions (cdfs) of PIE-D, defined in (3), are shown in Fig. 4. Under both launch conditions, the average-mode method usually generates larger PIE-D values, especially with a large offset in the link. However, if we consider a coverage of $99 \%$, the penalty difference predicted by these two method is $0.7 \mathrm{~dB}$ for a $7-\mu \mathrm{m}$ offset for both the center launch and the offset launch and less than $0.1 \mathrm{~dB}$ for a $5-\mu \mathrm{m}$ offset.

\section{CONCLUSION}

In this letter, we investigate the statistics of EDC performance, measured by PIE-D, of 300-m MMF links. We calculated the channel responses using both the individual-mode method and the average-mode method. We found a strong dependence of the PIE-D value on the channel model that was used, especially when there was a connector with a large offset in the link.

In general, the average-mode method predicts a larger EDC penalty. However, if one considers a coverage of $99 \%$ with moderate offsets $(5 \mu \mathrm{m})$ in the link, the EDC penalties predicted by both methods are close. In order to obtain the statistics of the EDC performance in installed fiber links, one should choose the appropriate fiber model, taking into account the length of the fiber, the magnitude of offset, and the percentage of coverage. In addition, further investigation of the EDC penalty with a finite number of taps is needed.

\section{ACKNOWLEDGMENT}

The authors thank the participants in the IEEE 802.3 aq task force for fruitful discussions.

\section{REFERENCES}

[1] L. Thon, P. Dawe, J. Jaeger, and A. V. Schyndel, "Serial EDC proposal for 10GBASE-LRM working document," in IEEE 802.3 aq March 2005 Plenary Meeting [Online]. Available: http://www.ieee802.org/ 3/aq/public/jul04/lawton_3_0704.pdf

[2] J. P. Weem, P. Kirkpatrick, and J.-M. Verdiell, "Electronic dispersion compensation for 10 Gigabit communication links over FDDI legacy multimode fiber," in Tech. Dig. Optical Fiber Communication Conf. OFC/NFOEC 2005, vol. 5, Paper OFO4.

[3] J. R. Barry, E. A. Lee, and D. G. Messerschmitt, Digital Communication, 3rd ed. New York: Springer, 2003.

[4] Y. Sun, P. Hallemeier, H. Ereifej, O. V. Sinkin, B. S. Marks, and C. R. Menyuk, "Experimental and theoretical study of a $10 \mathrm{~Gb} / \mathrm{s}$ multimode fiber link with connection offsets," presented at the CLEO/QELS 2005, Baltimore, MD, Paper CWG4.

[5] P. Pepeljugoski, S. Golowich, A. J. Ritger, P. Kolesar, and A. Risteski, "Modeling and simulaton of next generation multimode fiber links," $J$. Lightw. Technol., vol. 21, no. 5, pp. 1242-1255, May 2003.

[6] P. Pepeljugoski, M. Hackert, J. Abbott, S. Swanson, S. Golowich, A. J. Ritger, P. Kolesar, Y. C. Chen, and P. Pleunis, "Development of system specification for laser-optimized $50 \mu \mathrm{m}$ multimode fiber for multigigabit short-wavelength LANs," J. Lightw. Technol., vol. 21, no. 5, pp. 1256-1274, May 2003.

[7] K. Petermann, "Nonlinear distortions and noise in optical communication systems due to fiber connector," IEEE J. Quantum Electron., vol. QE 16, no. 7, pp. 761-770, Jul. 1980.

[8] G. Papen and G. Murphy, "Modal noise in multimode fibers under restricted launch conditions," J. Lightw. Technol., vol. 17, no. 5, pp. 817-822, May 1999.

[9] A. W. Snyder and J. D. Love, Optical Waveguide Theory. Norwell, MA: Kluwer, 2000

[10] G. Shaulov and Y. Sun, "DMD simulations based on scaled/non-scaled index profiles and comparison with Cambridge model results," in IEEE 802.3 aq Sep. 2004 Interim Meeting [Online]. Available: http://www. ieee802.org/3/aq/public/sep04/shaulov_1_0904.pdf

[11] K. Nawata, "Multimode and single-mode fiber connectors technology," IEEE J. Quantum Electron., vol. QE-16, no. 6, pp. 618-627, Jun. 1980.

[12] M. Webster, L. Raddatz, I. H. White, and D. G. Cunningham, "A statistical analysis of conditioned launch for Gigabit Ethernet links using multimode fiber," J. Lightw. Technol., vol. 17, no. 9, pp. 1532-1541, Sep. 1999.

[13] P. Dawe, L. Aronson, J. McVey, S. Bhoja, J. Ewen, M. Lobel, P. Popescu, A. Shanbhag, N. Weiner, and B. Willcocks, "How many connections for 10GBASE-LRM?," in IEEE 802.3 aq March 2005 Plenary Meeting [Online]. Available: http://grouper.ieee.org/groups/ 802/3/aq/public/mar05/dawe_1_0305.pdf

[14] S. Bhoja, "Channel metrics for EDC-based 10GBASE-LRM," in IEEE 802.3 aq July 2004 Plenary Meetting [Online]. Available: http:// grouper.ieee.org/groups/802/3/aq/public/jul04/bhoja_1_0704.pdf 